\title{
A Proposal for the Theoretical Investigation of Light-Ion Beam Propagation Through a Residual Plasma Environment
}

\author{
Lester E. Thode \\ Erick L. Lindman
}
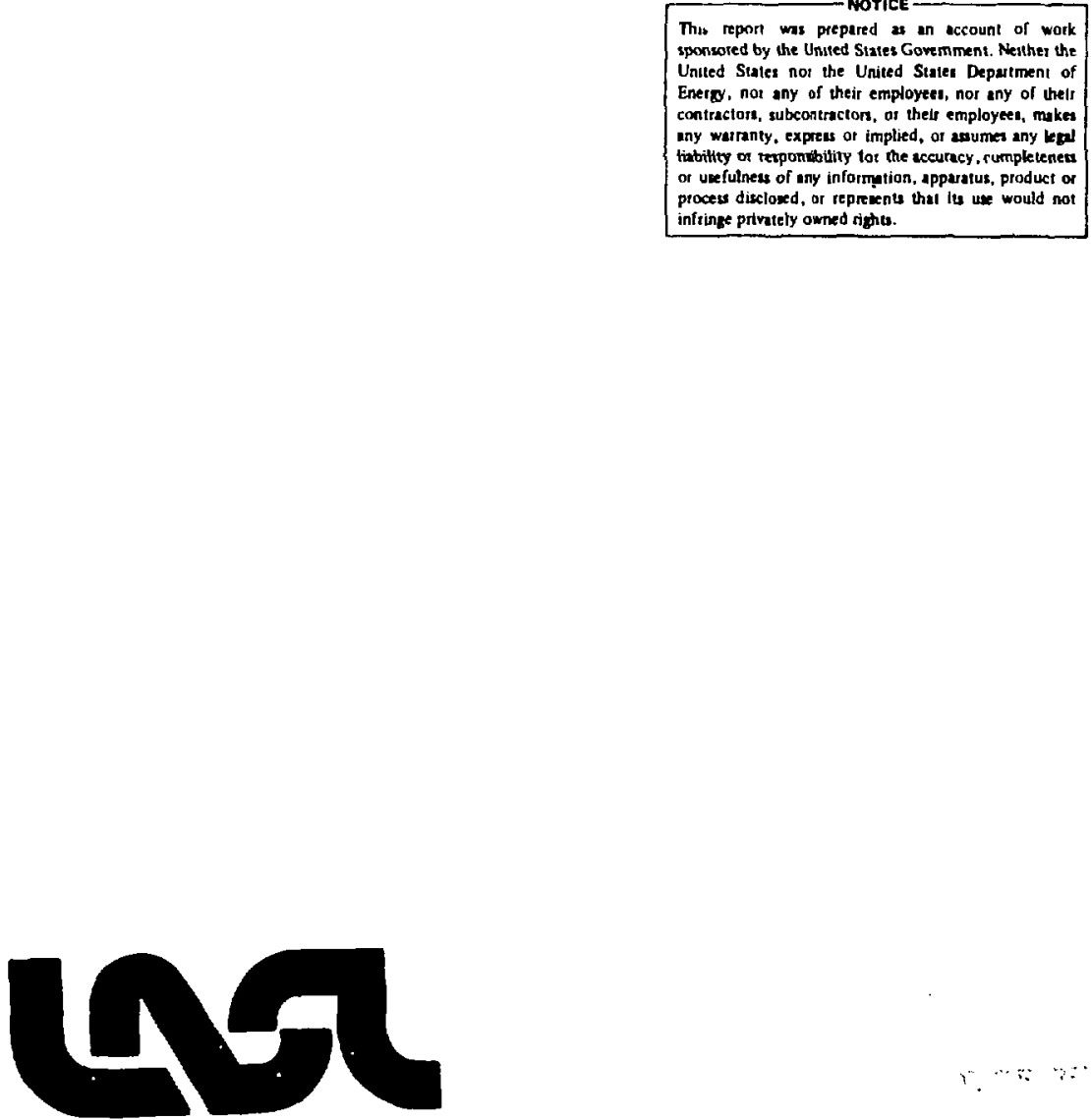


\title{
A PROPOSAL FOR THE THEORETICAL INVEST IGATION OF LIGHT-ION BEAM PROPAGATION THROUGH A RESIDUAL PLASMA ENVIRONMENT
}

\author{
by
}

Lester E. Thode and Erick L. Lindman

\begin{abstract}
In collaboration with the TRW Defense and Space Systems Group, a 22-month investigation of ion beam propagation in residual plasma is proposed by the Los Alamos Scientific Laboratory. We hope to gain insight into the nonlinear physics of microinstabilities associated with a well-collimated ion beam propagating through a plasma, to isolate parameter regimes where problem areas for propagation appear to exist, and to suggest techniques to overcome any problems. A strong interplay between theory and two-dimensional, electromagnetic particle-in-cell simulation is the key to the investigation.
\end{abstract}

\section{SCOPE}

A joint project to determine the feasibility of a light-ion driver for inertially confined fusion is proposed by TRH and Los Alaros Scientific Laboratory (LASL). The project involves the use of a high-current accelerator developed by TRW $^{1}$ to produce a well-collimated light-ion beam that is compressed in space and time during transport through a residual plasma to a pellet.

Although the net current of the proposed accelerator is large, preliminary analysis indicates that the initial accelerator design can be carried out by TRW using an electrostatic code. ${ }^{1}$ In fact, using a tine-dependent, electromagnetic code for initial accelerator design appears unjustified at this time because the presently unknown effects of microinstabilities on light-ion beam propagation could strongiy impact accelerator design. In collaboration with TRW, we propose to study tre fundamental physics associated with well-collimated ion beam neutralization and stability. 
A variety of microinstabilities can occur as an ion beam propagates through a plasma: the ion beam-electron two-stream instability, the neutralizing electron beam-electron two-stream instability, the ion beam-ion two-stream instability, and the Weibel instability. Such microinstabilities are driven by the free energy associated with the directed beam energy. The strength of the instabilities depends $w_{i}$ on both beam and plasma parameters and the technique of beam compression and neutralization.

A strong interplay between theory and two-dimensional, electromagnetic particle-in-cell simulation will be the key to the investigation. The LASL particle-in-cell code CCUBE obtains time- and space-dependent solutions in any orthogonal coordinate system. ${ }^{2}$ Moreover, CCUBE has been used excensively to study the class of microinstabilities we propose to investigate. ${ }^{3,4}$

Realizing both the power and limitations of a particle-in-cell simulation code, we hope to gain insight into the nonlinear physics of microinstabilities associated with a well-collimated ion beam propagating through a plasma. We also intend to isolate parameter regions where possible problem areas for propagation appear to exist and suggest techniques to overcome any problems. Considering preliminary desjgn of code runs and cost, it would not be possible to simulate the exact length and time scales relevant to the TRW concept. Thus, only qualitative answers concerning beam stability and neutralization should be expected from the simulations. But from the insight gained, quantitative solutions can then be obtained using hybrid-numerical and analytical techniques.

Although the proposed research is directed towards the TRW light-ion driver concept, it is sufficiently general to provide a stability assessment for most 1ight- or heavy-ion beam driver concepts. Of course, the propagation of current-neutralized light- or heavy-ion beams is insured by removing the residual plasma. Thus, the investigation is not rundamental to the demonstration of inertially confined controlled thermonuclear fusion, but is associaled with reactor considerations.

The 22-month investigation will begin 1 month after formal notification of funds is received. Reports will be prepared as required by the Department of Energy (DOE).

\section{I . MICROINSTABILITIES}

A problem associated with light- or heavy-ion-driven pellet implosion concepts is the stable propagation and focusing of the ion beam through a residual 
plasma resulting from a previous microexplosion. If the ion beam is sufficiently well collimated to achieve the required level of compression through ballistic focusing before interacting with the pellet, it appears that the beam is intrinsically unstable.

\section{A. Ion Beam-Electron Interaction}

Assuming a well-collimated ion beam propagating through an infinite, homogeneous plasma, the linear dispersion relation describing the interaction is 5,6 $\left(k^{2} i^{2}-w^{2}+w_{p}^{2}+\gamma_{b}^{-1} w_{b i}^{2}\right)\left[1-\frac{w_{e}^{2}}{w^{2}}-\frac{\gamma_{b}^{-3} u_{b i}^{2}}{\left(w-k_{n} v_{b}\right)^{2}}\right]-\frac{\gamma_{b}^{-1} w_{b i}^{2} w_{p}^{2} k_{\perp}^{2} v_{b}^{2}}{w^{2}\left(w-k_{z} v_{b}\right)^{2}}=0$,

where the electric field perturbation is

$$
E=\sum_{k} E_{k} \exp [i(\bar{k} \cdot \bar{x}-w t)]
$$

and $k^{2}=k_{1}^{2}+k_{z}^{2}$. We define the residual plasma frequency as $w_{p}=\left(w_{p i}^{2}+w_{p e}^{2}\right)^{1 / 2}$ with $w_{p i}$ and $w_{\text {pe }}$ being the plasma ion and electron frequencies, and $w_{b i}=\left(4 \pi \mathrm{b}_{i} z^{2} e^{2} / m_{i}\right)^{1 / 2}$ is the ion beam plasma frequency. In obtaining Eq. (1), the beam and plasma are assumed cold. The drift velocity of the beam $v_{b}$ is related to the relativistic factor $\gamma_{b}=\left[1-\left(v_{b} / c\right)^{2}\right]^{-1 / 2}$, where $c$ is the speed of 1 ight.

Equation (1) yields two instabilities. First, the electrostatic twostream instability initially grows with a temporal rate of 5,6

$$
\delta=\frac{\sqrt{3}}{2}\left(\frac{\omega_{\mathrm{bi}}^{2}}{2 w_{\mathrm{p}}^{2}}\right)^{1 / 3}\left[\frac{\left(k_{1}^{2} / \mathrm{k}^{2}\right)}{\gamma_{\mathrm{b}}}+\frac{\left(\mathrm{k}_{\mathrm{z}}^{2} / \mathrm{k}^{2}\right)}{\gamma_{\mathrm{b}}^{3}}\right]^{1 / 3} w_{\mathrm{p}}
$$

Second, the electromagnetic Weibel instability grows transverse to the beam at a temporal rate of 5,6

$$
\delta \cong \frac{v_{b}}{c}\left(\frac{w_{b i}}{w_{p} \gamma_{b}}\right)\left(\frac{k^{2} c^{2}}{k^{2} c^{2}+w_{p}^{2}}\right)^{1 / 2} w_{p}
$$


Equations (2) and (3) are valid near the injector where $w_{b i} \ll w_{p}$. For light ions $v_{b} / c \ll 1$ and $\gamma_{b} \cong 1$ and the electrostatic two-stream is expected to be the dominant instability near the injector.

Near the pellet the ion beam will have a large directional velocity spread caused by time compression, ${ }^{1}$

$$
\Delta v_{11} / v_{b} \sim 1 / 3 \quad
$$

which would be expected to reduce the strength of the instability. However, the effect of the longitudinal velocity spread Eq. (4) on the two-stream instability is somewhat compensated for because $w_{b i} \gtrsim w_{p}$ near the pellet. In fact, near the pellet the finite radius a of the beam may have to be taken into account if $a w_{p} / v_{b}<5$, in which case Eq. (1) is not strictly correct. Finally, near the pellet the Weibel instability may be competitive with the two-stream instability.

Near the injector the length $L_{I}$ over which a well-collimated light-ion beam might be disrupted by the two-stream instability is approximately

$$
L_{I} \cong 10\left(\frac{w_{p}}{w_{b i}}\right)^{2 / 3} \frac{v_{b}}{w_{p}} .
$$

Figure 1 shows the interaction length in centimeters as a function of $\mathrm{n}_{\mathrm{bi}} / \mathrm{n}_{\mathrm{pe}}$ for a $1-\mathrm{MeV}$ deuterium beam with $n_{b i} \cong 5 \times 10^{9} \mathrm{~cm}^{-3}$. Fiere $n_{p e}$ and $n_{b i}$ are the plasma electron particle density and ion beam particle density, respectively. Even if $w_{b i} \ll w_{p}$, the interaction length is quite short.

Based upon preliminary modeling of the nonlinear state of this instability, a well-collimated ion beam will relax in angle as well as energy. The model, known as the oblique approximation, ${ }^{7}$ has been extremely successful in predicting the two-dimensional relativistic electron beam-plasma interaction ${ }^{3,4}$ worked out by numerical simulation. To date, no two-dimensional simulations investigating the ion beam-residual plasma interaction have been carried out. If the ion beam does relax in angle, a violent disruption in beis focusing and transport occurs. Even if the model is incorrect and simulations indicate that this primary instability evolves in a one-dimensional fashion, defocusing resulting from over-neutralization of the beam might occur. This has been discussed by Sudan. ${ }^{8}$ 


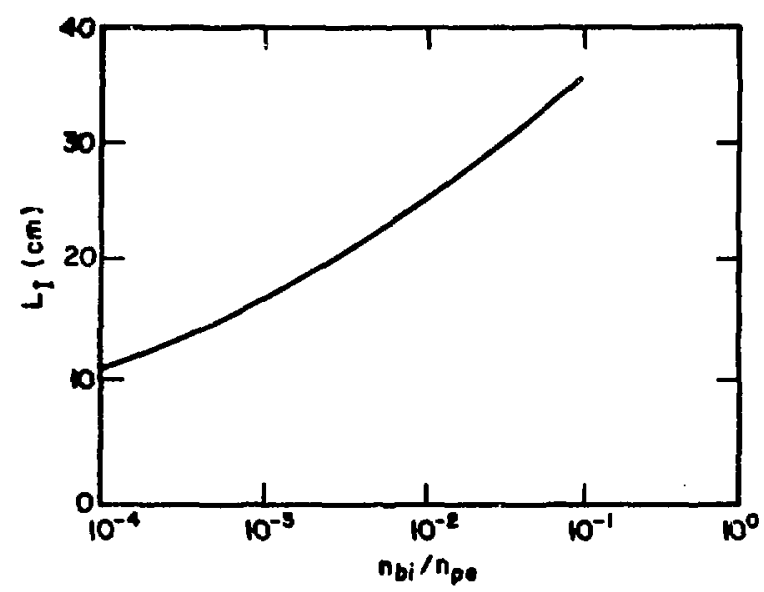

Fig. 1 .

Approximate interaction length for a $1-\mathrm{MeV}$, deuterium ion beam.

Near the injector the evolution of the two-stream instability might be affected by electron-ion collisions. Electron-ion collisions result in a reduction in the strength of the nonlinear state and increased interaction length. 9 However, it is required that

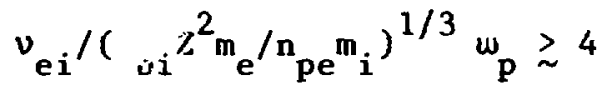

to observe a significant reduction of the interaction strength. For the typical residual plasma of $\mathrm{T}_{\mathrm{pe}} \cong(1$ is 3$) \mathrm{eV}$ and $\mathrm{n}_{\mathrm{pe}} \cong\left(10^{12}\right.$ to $\left.10^{14}\right) \mathrm{cm}^{-3}$, the electron-ion collision rate is

$$
v_{\text {ei }} \cong \frac{\ln \Lambda}{\wedge} w_{p e} \cong(1 \text { to } 50) \times 10^{-4} w_{\text {pe }}
$$

where $\wedge \cong 1.5 \times 10^{10}\left(\mathrm{~T}_{\mathrm{pe}}^{3 / 2} / \mathrm{n}_{\mathrm{pe}} \mathrm{e}^{1 / 2} \mathrm{)} \mathrm{eV}^{-\mathrm{cm}^{3 / 2}}\right.$. Thus, near the injector electronion collisions would retard the instability. But neac the pellet Eq. (6) is not satisfied, and collisions cannot be expected to reduce the instability in this region.

Experience indicates that inhomogenefty in the residual plasma particle density, although large, will not significantly reduce instabilities.

Note that the advantage of a well-collimated, low-charge state, heavy-ion beam suggested by Eqs̀. (5) and (6) is dubious because of stripping. In fact, light-ion beams may be at an advantage with respect to propagation since the nonlinear strength of the two-stream instability is weak relative to that of heavy-ion beams. 


\section{B. Neutralizing Electron Beam-Electron Interaction}

The primary ion beam-residual plasma streaming instability discussed previously can be stabilized by current-neutralizing the ion beam, as the high mobility of the neutralizing electrons can short any organized ion motion. However, simple linear theory suggests that the neutralizing electron beam is itself unstable. The electrostatic dispersion relation is

$$
1-\frac{w_{p i}^{2}}{w^{2}}-\frac{w_{p e}^{2}}{u^{2}}-\frac{w_{b i}^{2}}{\left(w-\bar{k} \cdot \bar{v}_{b}\right)^{2}}-\frac{w_{b e}^{2}}{\left(w-\bar{k} \cdot \bar{v}_{b}\right)^{2}}=0 \text {, }
$$

where $w_{b e} \gg w_{b i}$ is the plasma frequency of the neutralizing electron beam. For the well-collimated neutralizing electron beam assumed in obtaining Eq. (7), the instability is rather insensitive to electron-ion collisions and to gradients. Although this secondary instability probably would not disrupt the ion beam directly, it could lead to both loss of current neutralization, with the ion beam again becoming unstable downstream of the pcsition of the secondary instability, and a slight disruption of the ion beam as it passes through the position of the secondary instability caused by local current overneutralization.

As the interaction length $\mathrm{L}_{E}$ of this secondary instability is very short,

$$
L_{E} \cong 10 \gamma_{b}\left(\frac{n_{p e}}{n_{b i}}\right)^{1 / 3} \frac{v_{b}}{w_{p}},
$$

it could lead to continual loss of stripped electrons in a heavy-ion beam. Here the stripping occurs by classical processes as the residual plasma density is increased in order to increase $v_{e i}$.

Under these conditions the interaction length $L_{I}$ for a heavy-ion beam is comparable to that of a light-ion beam, but the nonlinear strength of the two-stream instability is larger for the heavy-ion beam. Thus, heavy-ion beams do not appear to have any distinct advantage with respect to stable transport over 1ight-ion beams.

Because the effect of the secondary electron-electron instability on current-neutralizied ion beam propagation has not been considered previously, we have performed two parti-le-in-cell simulations to clarify some questions concerning the nonlinear state of the instability. Both simulations were 
carried out using the two-dimensional, electromagnetic code CCUBE. We considered the interaction of a well-collimated light- and heavy-ion current-neutralized beam. The major question was the evolution of the neutralizing electron beam-electron instability in the presence of the ion beam. The parameters for the heavy-ion beam run were obtained from an existing relativistic electron beam-plasma simulation to facilitate corparison. For the light-ion beam simulation, the parameters were chosen to represent the TRW concept. We wish to emphasize that both simulations are the first of their kind, being exploratory in character, and are intended only to give some initial insight. Our basic conclusion is that the secondary neutralizing electron beam-electron instability appears as a potential problem and must be investigated in detail, both for light- and heavy-ion beam propagation.

In Fig. 2, the geometry of the light-ion beam simulation is shown. A current-neutralized $5-\mathrm{MeV}$ deuterium beam is injected into a homogeneous plasma with $n_{b e}=10 n_{p e}$ and $n_{b e}=n_{b i}$. The actual simulation was carried out in a coordinate system defined by

$$
x 1=z m_{b e} / c
$$

and

$$
\mathrm{X} 2=\left(\mathrm{aube}_{\mathrm{be}} / \mathrm{c}\right) \ln (1+\mathrm{r} / \mathrm{a}),
$$

where $r$ and $z$ are cylindrical coordinates. Azimuthal symmetry is assumed in space. Particles are absorbed on all surfaces and reflected on the axis. The axial field boundary conditions are open ended and the radial wall is a perfect conductor. Initially, the plasma electron temperature is $\mathrm{T}_{\mathrm{pe}} \cong 3.2 \mathrm{eV}$.

The early evolution of the neutralizing electron beam-electron instability proceeds as predicted by Eq. (1). As expected, saturation of the instability occurs by the trapping of plasma electrons; see Fig. 3. Plasma density gradient formation is observed as a result of energy transfer from the neutralizing electron beam to the plasma electrons. Such a gradient increases the deposition length and tends to retard, but not stabilize, the instability. The nonlinear strength of the instability is weak since $w_{b e}>w_{\text {pe }}$, consistent with relativistic electron beam experiments. 10 Plasma electrons are accelerated up to the 
ion beam velocity during instability, thus helping to preserve current neutralization.

The basic geometry used for the heavy-ion simulation is shown in Fig. 4. A 350-keV current-neutralizing electron beam is injected together with the ion beam into a plasma with $n_{p e}=10 \mathrm{n}_{\mathrm{be}}$ and $\mathrm{n}_{\mathrm{be}}=\mathrm{n}_{\mathrm{bi}}$. Boundary conditions are the same as for the light-ion simulation, and transformation Eqs. (9) and (10) are used. For this case, the neutralizing electron beam-electron two-stream interaction develops at $t z \cong 15 \mathrm{c} / w_{p}$ and evolves essentially independently of the ion beam. Because the neutralizing electron beam is relativistic in the heavy-ion simulation, the interaction is strong and two-dimensional. For example, the neutralizing electron beam configuration space is shown in Fig. 5 . The instability is coupling $40 \%$ of the evergy from the neutralizing electron beam to the plasma electrons. Under these conditions, a loss of current neutralizaticn occurs in the downstream region. Also, as the ion beam leaves the plasma edge at $z=70 \mathrm{c} / w_{p}$, it is unable to electrostatically current neutralize itself again. A slight random motion is imparted to the ion beam in the instability region.

On the basis of this simulation, the secondary electron-electron instability may pose a potential problem for heavy-ion bean propagation and should be investigated in detail. Our simple estimates of the strength of the instability indicate that it is difficult to mitigate. However, the simulation probably overemphasizes the effect of the instability because all the stripped electrons would not appear instantaneously.

C. Neutralization Considerations

Control of the secondary neutralizing electron beam-electron instability appears to depend primarily upon the neutralizing electron beam temperature, which in turn depends upon the technique of ion beam neutralization and the finite radial size of the beam. If an accelerating grid is used to produce the neutralizing electron beam, we expect the neutralizing beam to be well collimated and thus more susceptible to instability. On the other hand, electrostatic current neutralization carried out by passing the light-ion beam through a localized plasma or electron cloud and vacuum interface might produce a thermal neutral beam distribution. However, the violent character of the neutralization may lead to some internal pressure build-up within the ion beam, which might ultimately limit compression. Some investigation into the latter technique of neutralization appeirs justified. 


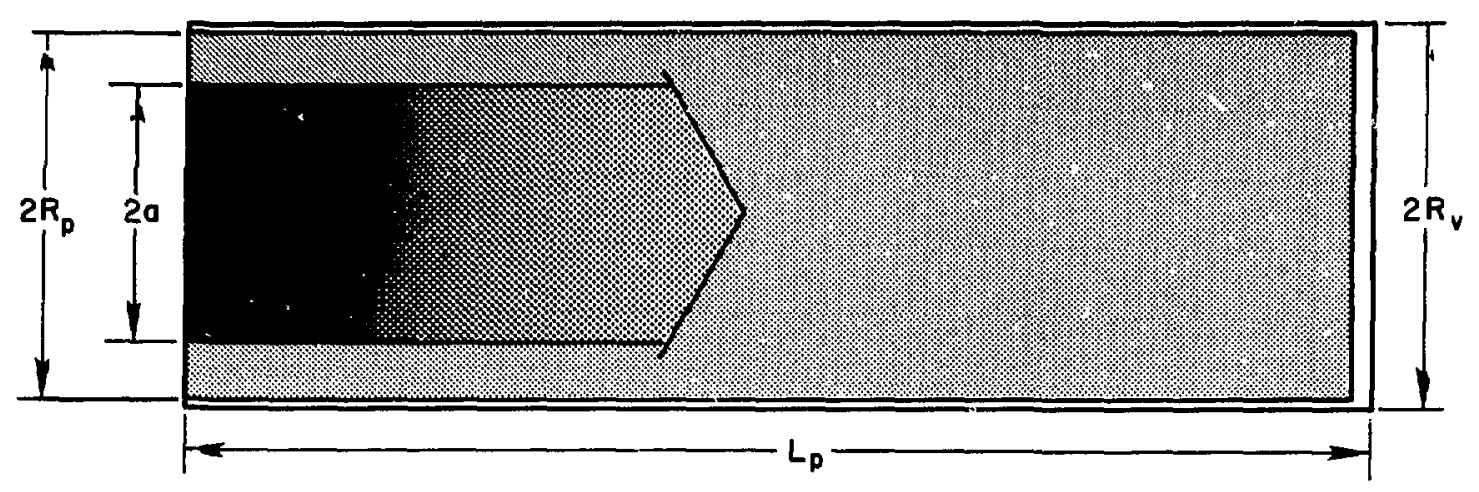

Fig. 2. Geometry for light-ion particle-in-cell simulation. A $1.36-k e V$ current-neutralizing electron beam and associated ion beam with $\mathrm{m}_{i} / \mathrm{m}_{\mathrm{f}}=3670.5$ is injected into a 3.2-eV plasma with $n_{h i} 9 n_{p e}=10.0$. Dimensions are $a=15 \mathrm{c} / w_{\text {pe }}, R_{p}=38 \mathrm{c} / w_{p e}, R_{y}=40 \mathrm{c} / w_{p e}$, and $L_{1}=3.5 \mathrm{c} / w_{\text {pe' }}$ Initially, bbe' the beams ahd the plasma have pe constarit density.

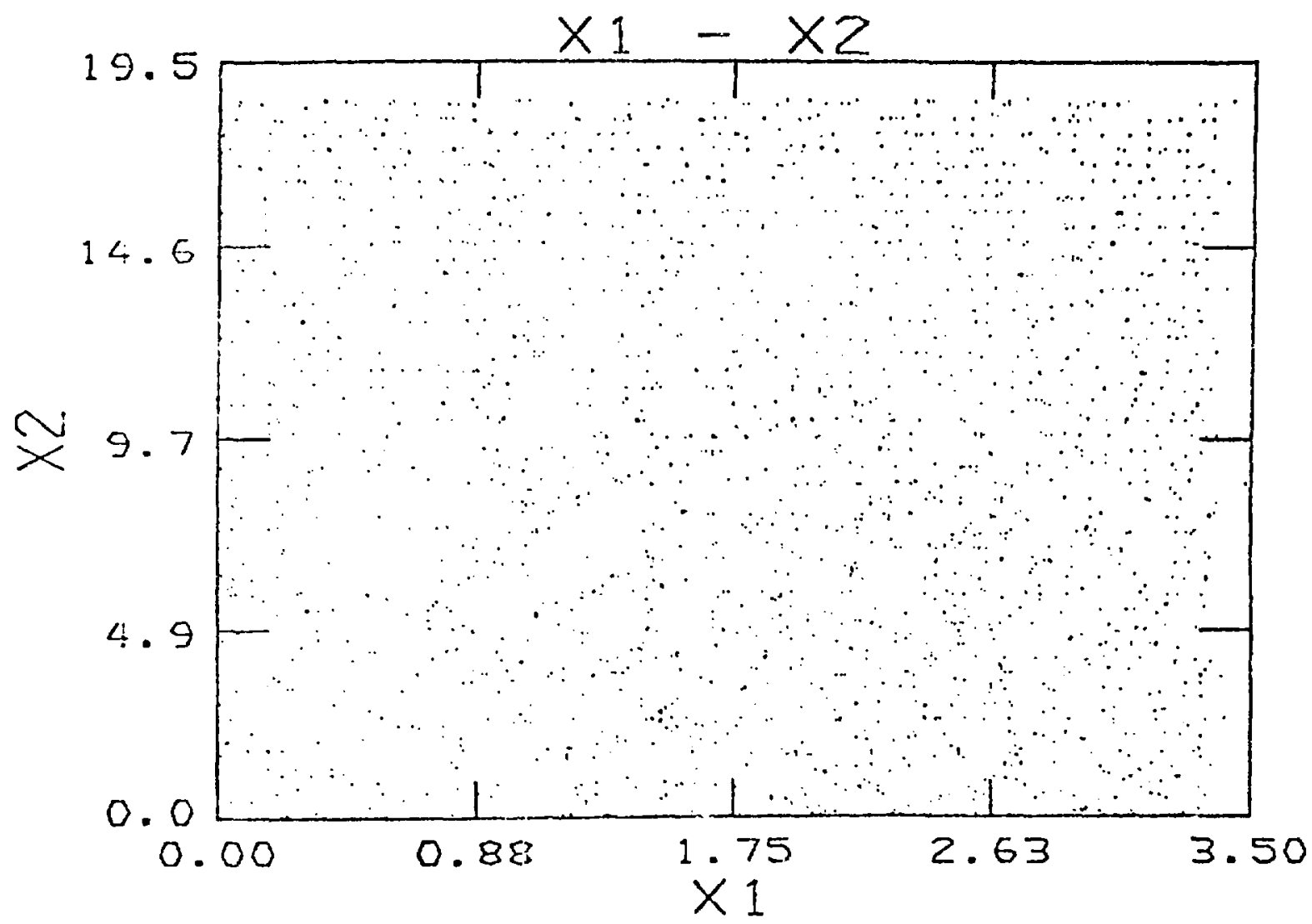

Fig. 3. Configuration space of plasma electrons in the transformed cooidinate system defined by Eqs. (9) and (10). Note vortex structure indicating trapping as the saturation mechanism. 


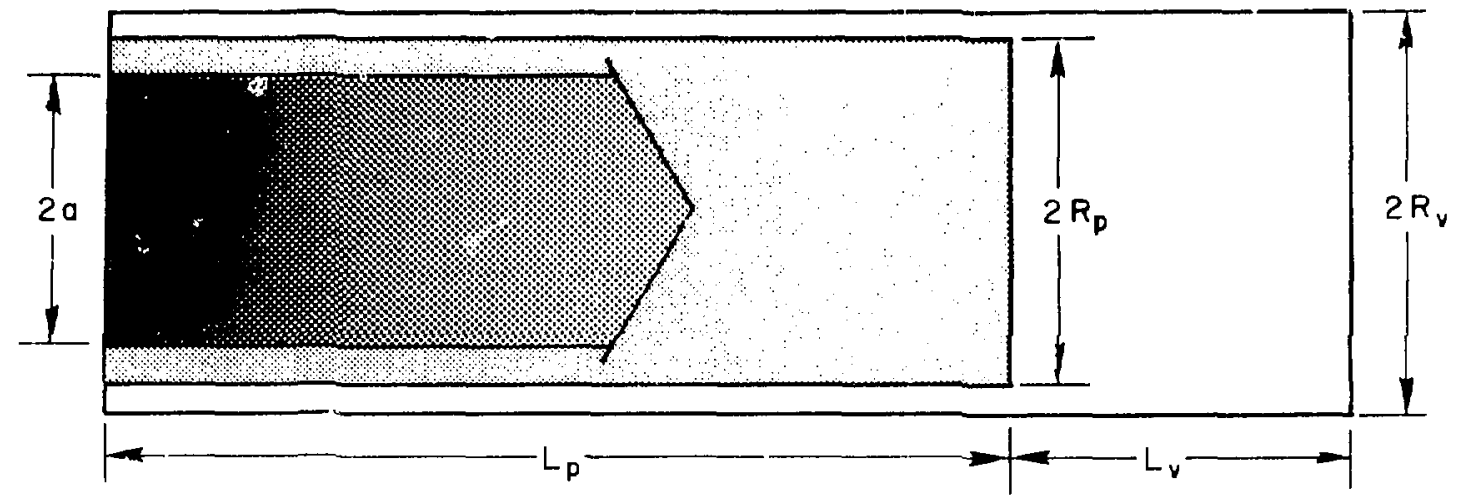

Fig. 4. lienmetry tor heavy-ion particle-interll simulation.

A is)-keV curaent-mentralizing electron heam and

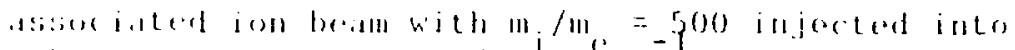

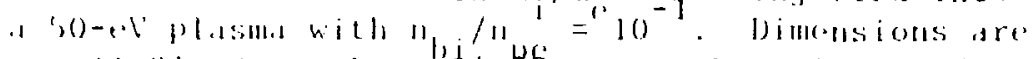

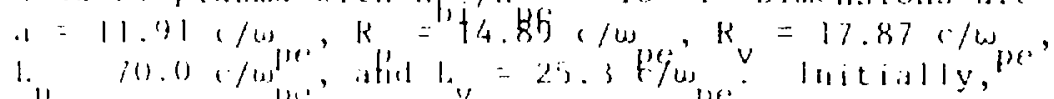

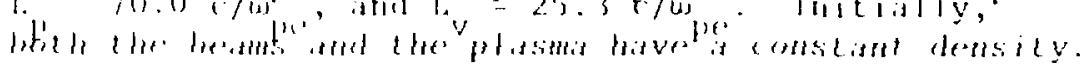

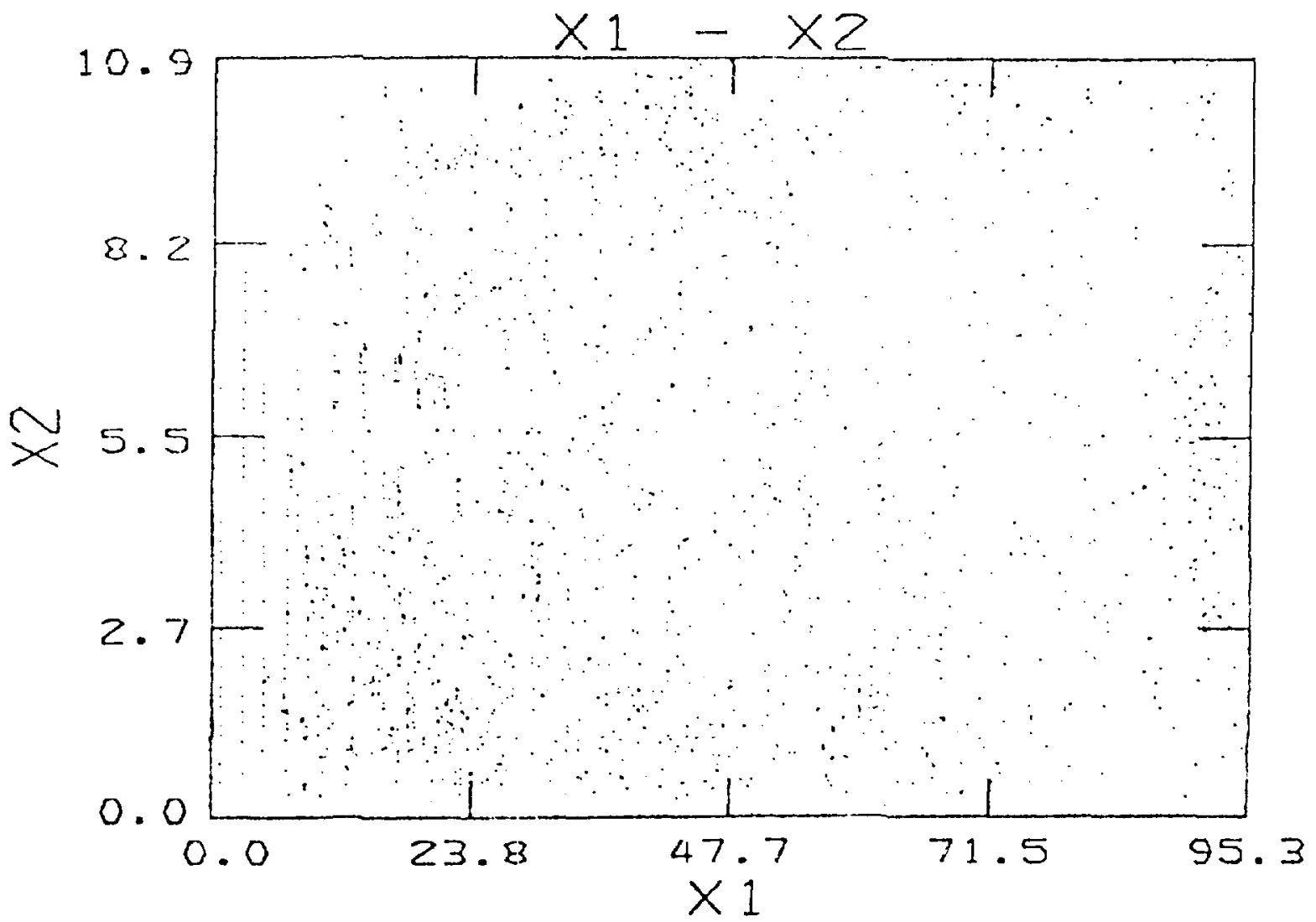

Fig. 5. Configuration space of neutralizing beam electrons in the transformed cooldinate system defined by ligs. (9) and (10). Note the strong two-dimensional resaxation of the electrons. 
D. Ion Beam-Ion Instability

If the electron temperatures are included in the fluid approximation, the electrostatic dispersion relation becomes

$$
1-\frac{w_{p e}^{2}}{w^{2}-k^{2} \theta_{p e}^{2}}-\frac{w_{p i}^{2}}{w^{2}}-\frac{w_{b e}^{2}}{\left(w-\bar{k} \cdot \bar{v}_{b}\right)^{2}-k^{2} \theta_{b e}^{2}}-\frac{w_{b i}^{2}}{\left(w-\bar{k} \cdot \bar{v}_{b}\right)^{2}}=0,
$$

where $\theta_{p e}$ and $\theta_{\text {be }}$ are the plasma electron and beam electron temperatures, respectively. Consider the limits $u \ll k \theta_{\text {pe }}$ and $w-\bar{k} \cdot \bar{v}_{b} \ll k \theta_{b e}$. Defining $\lambda_{D}$ as the effective electron Debye length for the system, Eq. (11) becomes

$$
1+\frac{1}{k^{2} \lambda_{D}^{2}}-\frac{w_{p i}^{2}}{w^{2}}-\frac{w_{b i}^{2}}{\left(w-\bar{k} \cdot \bar{v}_{b}\right)^{2}}=0
$$

Assuming the growth rate $\delta$ is small relative to $\omega_{0}=k \lambda_{D} w_{p i} /\left(1+k^{2} \lambda_{D}^{2}\right)^{1 / 2}$, Eq. (12) yields

$$
\delta=\frac{\sqrt{ } 3}{2}\left(\begin{array}{l}
m_{e} \\
m_{i}
\end{array}\right)^{1 / 2}\left(\frac{n_{b i}}{2 n_{p e}}\right)^{1 / 2}\left[\frac{k \lambda_{D}}{\left(1+k^{2} \lambda_{D}^{2}\right)^{1 / 2}}\right] \omega_{p e}
$$

This growth rate is down by a factor of only $\left(m_{e} / m_{i}\right)^{1 / 6}$ from the electron-ion acoustic instability and is strongly transverse to the beam for the parameters of interest.

Simulations ${ }^{11}$ with a single Maxwellian electron distribution and equaldensity ion beams have shown that the instability is stabilized by ion Landau damping when the transverse temperature of the ion beam rises to

$$
T_{b i} \cong \frac{1}{4} T_{e}
$$

where $T_{e}$ is the electron temperature for the single Maxwellian. Assuming this result is applicable to more general distributions, the transverse ion velocity spread $v_{\perp}$ required for stability divided by the beam velocity is 


$$
\frac{v_{1}}{v_{b}}=\left(\frac{T_{e}}{4 E_{b i}}\right)^{1 / 2} \sim 1.5 \times 10^{-3},
$$

where $E_{b i}$ is the iun beam energy. If focusing to $1 \mathrm{mn}$ from $10 \mathrm{~m}$ away is desired, the transyerse velocity must satisfy

$$
\frac{v_{1}}{v_{b}} \sim 10^{-4} \text {, }
$$

and this instability may prevent the desired focusing.

III. REACTOR APPLICATIONS AND RELATION TO OTHER PROJECTS

The proposed theoretical program is directly applicable to inertial confinement fusion reactors using a light- or heavy-ion beam as a driver.

On a more general level, the research is related to relativistic electron beam propagation through partially ionized gas, relativistic elect:on beam heating of high-density plasma, and the high-altitude "pickup" problem (Appendix). To a lesser extent, the analysis may have some bearing on beam interactions with blowoff plasma for electron-beam-driven inertial confinement fusion and the hot-electron problem associated with laser-driven inertial confinement fusion.

IV. INTENSE PARTICLE BEAM THEORY PUBLICATIONS

The Intense Particle Beam Theory Group ( $T-15)$ at LASL primarily investigates the coherent collective nature of particle beams. Topics of incerest are collective-ion acceleration, beam propagation, high-density plasma heating, beam coupling to periodic structures, and diode development. The group is also involved in the development of numerical methods for plasma simulation. Publications by T-15 during FY78 are:

1. Rickey J. Faehl, Brendan B. Godfrey, and William R. Shanahan, "Simulation of Nonlinear Cyclotron Waves in an Inhomogeneous Magnetic Field," Bull. Am. Phys. Soc. 23, 589 (1978). 
2. R. J. Faehl, B. S. Newberger, B. B. Godfrey, and W. R. Shanahan, "Sirnulation of Cyclotron Wave Growth in a Helical S.w:" Wave Structure," Bull. Am. Phy. Soc. 22, 1109 (1977).

3. N. F. Roderick, ${ }^{*}$ T. W. Hussey, ${ }^{*}$ R. J. Faehl, and R. W. Boyd, $\stackrel{*}{*}$ "TwoDimensional Simulation of the Hydromagnetic Rayleigh-Taylor Instability in an Imploding Fuil Piasma," Appl. Phys. Lett. 32, 273 (1978).

4. R. J. Faehl and N. F. Roderick," "Intensity Dependence of Inverse Bremsstrahlung Absorption in an Inhomogeneous Standing Wave," Phys. Fluids 21, $793(1978)$.

5. R. J. Faehl, W. R. Shanahan, and B. B. Godfrey, "Nonlinear Characteristics of Cyclotron Waves in an ARA Configuration," to be published in the Proc. of the 3rd Int. Conf. on Collective Methods of Acceleration, Laguna Beach, California, May 22-25, 1978 (IEEE, New York, NY).

6. B. B. Godfrey, "Numerical Simulation of Autoresonant Ion Acceleration," IEEE Trans. Plasma Sci. 5, 223 (1977).

7. B. B. Godfrey, R. J. Faehl, B. S. Newberger, W. R. Shanahan, and L. E. Thode, "Collective Ion Acceleration, July 1976 - September 1977," Los Alamos Scientific Laboratory report LA-7148-PR (February 1978).

8. Brendan B. Godfrey, Rickey J. Faehl, and William R. Shanahan, "Adiabatic Variation of Linear Wave Amplitudes in Autoresonant Collective Ion Acceleration," Bull. Am. Phys. Soc. 23, 589 (1978).

9. Brendan B. Godfrey, "Application of Galerkin's Method to Particle-in-Cell Plasma Simulation," Proc. of the Eighth Conf. on Numerical Simulations of Plasmas, Monterey, California, May 15-17, 1978 (IEEE, New York, NY).

10. B. B. Godfrey, R. J. Faehl, B. S. Newberger, W. R. Shanahan, and L. E. Thode, "Relativistic Electron Beam Cyclotron Wave Growth in Helical Slow Wave Structures," 2nd Int. Conf. on High Power Electron and Ion Beam Research and Technology (Ithaca, New York, 1977), Vol. II, p. 541.

11. B. B. Godfrey, "The Localized Pinch Model as a High Energy Collective Ion Acceleration Mechanism," to be published in IEEE Trans. Plasma Sci.

12. B. B. Godfrey and B. S. Newberger, "Wave Amplitude Variation and Energy Flow in Autoresonant Collective Ion Acceleration, "to be published in J. App 1. Phys.

13. B. B. Godfrey, R. J. Faehl, and W. R. Shanahan, "Collective Ion Acceleration, October 1977 - September 1978," to be published as a Los Alamos Scientific Laboratory report.

*Now at AFWL, Kirtland AFB.

**Now at Headquarters USAF, Washington, D.C. 
14. Michael E. Jones and J. Fukai," "Evolutior: of the Explosive Inslability in a Simulated Beam-Plasma," Bull. Am. Yhys. Soc. 22, 1176 (1977).

15. B. S. Newberger, R. J. Faeh1, B. B. Godfrey, and W. R. Shanahan, "EndEffects in Cyclotron Wave Growth in a Helical Slow Wave Structure," Bull. Am. Phys. Soc. 22, 1109 (1977).

16. Barry S. Newberger, "Relativistic Electron Beam Propagation in Low Density Neutral Gas," in the Conf. Record of the 1978 IEEE Int. Conf. on Plasma Science, Monterey, California, May 15-17, 1978, (IEEE, New York, NY) p. 227.

17. W. R. Shanahan, B. B. Godfrey, and R. J. Faeh1, "Slow Cyclotron Wave Growth by Periodic Inductive Structures," to be published in Proc. of the 3rd Int. Conf. on Collective Methods of Acceleration, Laguna Beach, California, May 22-25, 1978 (IEEE, New York, NY).

18. W. R. Shanahan, "Multidimensional Effects in the Inhomogeneous Beam-Plasma Instability," Phyis. Fluids 21, 000 (1978).

19. Lester E. Thode, "Effect of Electron-Ion Collisions on the Nonlinear State of the Relativistic Two-Stream Instability," Phys. Fluids 20, 2121 (1977).

20. Lester E. Thode, "Relativistic Streaming Instability Enhanced by Judicious Choice of External Magnetic Field Strength," in the Conference Record of the 1978 IEEE Int. Conf. on Plasma Science, Monterey, California, (IEEE, New York, NY) p. 284.

21. Lester E. Thode, "Proposal for Study of Vacuum Adiabatic Compression of a Relativistic Electron Beam Generated by a Foilless Diode," Los Alamos Scientific Laboratory report LA-7169-P (February 1978).

22. Lester E. Thode, "Preliminary Invesfigatiogo of Anomalous Relativistic Electron Beam Deposition into a $10^{17}$ to $10^{20} \mathrm{~cm}^{-3}$ Density Plasma," Los Alamos Scientific Laboratory report LA-7215-MS (April 1978).

23. Lester E. Thode, "Design Considerations for the Air Force Weapons Laboratory FX25 Plasma Heating Experiment," Los Alamos Scientific Laboratory report LA-7233-MS (April 1978).

24. Lester E. Thode, "Proposal for the Theoretical Investigation of the Relativistic Beam-Plasma Interaction with Application to the Proof-of-Principle Electron Beam-Heated Linear Solenoidal Reactor," Los Alamos Scientific Laboratory report IA-7359-P (September 1978).

25. Lester E. Thode, Brendan B. Godfrey, and William R. Shanahan, "Vacuum Propagation of Solid Kelativistic Electron Beams: Correlations Among Experiment, Simulation, and Theory," to be published in Phys. Fluids.

*J. Fukai is at Auburn University, Auburn, Alabama. 


\section{PROPOSEI INVESTIGATION FOR FIRST 10 MONTHS}

During the first 10 months of the investigation, we intend to broadly scope light-ion beam neutralization and stability in a plasma. Our aim is to isolate any critical problem area that might exist for the TRW concept.

In order to understand the process of neutralization, several two-dimensional particle-in-cell simulations of well-collimated light-ion beam injection into a plasma and an electron cloud are planned. Because the propagation velocity of a light-ion beam is low, it may be possible for the beam to pick up electrons as it passes through a plasma/vacuum interface. This technique might produce a high-temperature neutralizing electron beam, thus reducing the electron beam-electron two-stream instability.

Because the actual effect of the ion beam-electron plasma two-stream instability is unknown, several two-dimensional particle-in-cell simulations investigating the instability near the injector are planned. In addition, the linear theory of the ion beam-electron two-stream instability, the ion beam-ion two-stream instability, and Weibel instability in a collisional plasma will be investigated.

Linear theory of a well-collimated ion beam with a co-moving equal-density electron beam will be carried out, including collisions and electron temperature. Insight into the neutralizing electron beam temperature will have been obtained from particle-in-cell simulations investigating neutralization. Twodimensional particle-in-cell simulations to determine if any of the secondary instabilities would lead to destruction of the current-neutralizing electron beam will be carried out.

VI . PROPOSED INVESTIGATION FOR THE SECOND YEAR

Realistically, the area of investigation in the second year will depend heavily upon the results of our first 10-month analysis of light-ion beam propagation. Generally, a much more detailed study of the nonlinear evolution in a collisional plasma of any instability that appears to be detrimental to propagation is envisioned. This would include two-dimensional particle-incell simulations, partial numerical simulations, and quasi-linear analysis. Estimates for the effect of ionization, gradients, and finite boundaries would be included. 
APPENDIX

THE "PICKUP" PROBLEM AND ITS RELATION TO ION BEAM

TRANSPORT IN INERTIALLY CONFINED FUSIGN

During the early seventies, a problem of interest to Anti-Ballistic Missile System designers involved a beam of ions in a partially ionized gas. The beam was made up of debris from a nuclear weapon. And the propagation medium was the ionosphere or air, partially ionized by the radiation from the weapon. The problem was that of "pickup" at altitudes above $200 \mathrm{~km}$, where the debris could penetrate several debris masses of air before being stopped by collisional interactions. Specifically, could plasma instabilities produce a sufficiently strong interaction between the debris and the air to cause the air overrun by the debris to be picked up and carried along? If this occurred, conservation of momentum would require that the volume occupied by the debris would expand more slowly and stop at a smaller maximum radius. If true, the ability o. various missile detection systems to see incoming missiles would be strongly affected.

In this problem, as in the beam transport problem in ion-driven, inertially confined fusion, the various beam-plasma instabilities had to be examined individually to determine their influence on the system. The beam density varied from well above the background plasma density to well below it. Ionization levels and background electron temperatures also varied substantially. The high-altitude problem did differ, however, in that a magnetic field was present.

Several instabilities were considered and then discarded as possible contributions to a pickup effect because they produced a spread in transverse velocities rather than substantial effects on the longitudinal component. Among these was the electrostatic ion beam-ion plasma two-stream. Other instabilities such as the ion beam-electron plasma two-stream produced too weak an effect on the ions to cause a strong pickup effect.

Transverse spreads in velocity and weaker effects are, however, of great interest in the ion beam transport problem because ballistic focusing of an ion beam from $10 \mathrm{~m}$ to less than $1 \mathrm{~cm}$ in radius is required. The knowledge of the effects of beam-plasma instabilities obtained in the High Altitude Nuclear 
Weapons Eftects Program thus provides an excellent base from which this study can start. Much of the work in the High Altitude Program was carried out at LASL and the benefits of that work are available to the proposed investigation. In particular, E. Lindman worked on the high-altitude problem for several years at LASL and is quite familiar with the knowledge that was attained in the program.

\section{REFERENCES}

1. TRW Proposal 33876.000, "L.I.F.E. Light Ion Fusion Experiment Key Concept Validation Study," (July 1978).

2. Nicia Grant Cooper; compiler, "Theoreticai Division Annual Report July 1975 - September 1976," Los Alamos Scientific Laboratory report LA-6816-PR (1976), p. 148. CCUBE was designed and implemented by B. B. Godfrey.

3. L. E. Thode, "Preliminary Inuestigatign of Anomalous Relativistic Electron Beam Deposition into a $10^{17}$ to $10^{2} \mathrm{~cm}^{-3}$ Density Plasma," Los Alamos Scientific Laboratory report LA-7215-MS (April 1978).

4. L. E. Thode, "Relativistic Streaming Instability Enhanced by Judicious Choice of External Magnetic Field Strength," in Conference Record of the 1978 IEEE International Conference on Plasma Science, Monterey, California, May 15-17, 1978, (IEEE, New York, NY) p. 284.

5. D. W. Ignat and J. H. Hirshfield, "Radiation from the Two-Stream lnstability," Phys. Rev. A1, 872 (1970).

6. B. B. Godfrey, W. R. Shanahan, and L. E. Thode, "Linear Theory of a Cold Relativistic Beam Propagating Along an External Magnetic Field," Phys. Fluids 18, 346 (1975).

7. L. E. Thode, "Energy Lost by a Relativistic Electron Beam Due to Two-Stream Instability," Phys. Fluids 19, 305 (1976).

8. R. N. Sudan, "Propagation and Defocusing of Inteinse Ion Beams in a Background Plasma," Phys. Rev. Lett. 37, 1613 (1976).

9. L. E. Thode, "Effect of Electron-Ion Collisions on the Nonlinear State of the Relativistic Two-Stream Instability," Phys. Fluids 20, 2121 (1977).

10. C. A. Kapetanakos and D. A. Hammer, "Plasma Heating by an Intense Relativistic Electron Beam," Appl. Phys. Lett. 23, 17 (1973).

11. D. W. Forslund and C. R. Shonk, "Numerical Simulation of Electrostatic Counterstreaming Instabilities in Ion Beams," Phys. Rev. Lett. 25, 281 (1970). 